\title{
Ethical issues in training future literary translators
}

Gulnara M. Kassymova*,

Suggested Citation:

and Teaching 9

Abstract 
1. Introduction

2. Theoretical background 
3. Method

4. Potentials of Kazakhstani Translation Studies Department's Curriculum 
International Journal of Learning and Teaching 9 
5. Conclusion

\section{References}

The Interpreter and Translator trainer,

5 